\title{
Constructive Technology Assessment (CTA)
}

\section{Innovationsmitgestaltung als Prozess gesellschaftlicher Aufklärung und Erwartungsmoderation}

\section{Peter Stegmaier}

\section{Inhalt}

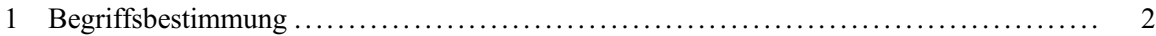

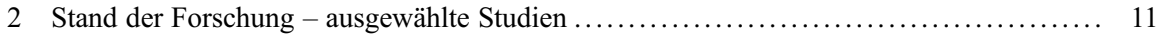

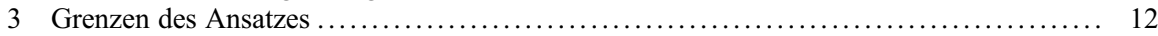

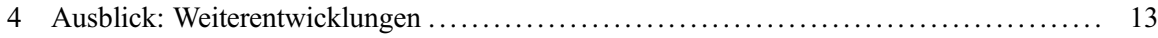

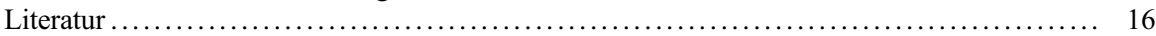

\section{Zusammenfassung}

Constructive Technology Assessment (CTA) ist eine Vorgehensweise für die vorausschauende Gestaltung von Technologien und anderen Innovationsbereichen. Solide Forschung im Vorfeld ist entscheidend für die Qualität der Interaktion mit den Teilnehmenden. Gegenstand von CTA-Interaktionen sind häufig Szenarien: hochverdichtete Narrative von sozio-technischen Konfigurationen, Innovationspfadkarten oder Innovationswertschöpfungsketten.

\section{Schlüsselwörter}

Constructive Technology Assessment · Constructive Innovation Assessment · Innovationsmitgestaltung $\cdot$ Antizipation $\cdot$ Szenarien

P. Stegmaier $(\bowtie)$

Department for Technology, Policy and Society (TPS), Section of Science, Technology and Policy Studies, University of Twente, Enschede, Niederlande

E-Mail: p.stegmaier@utwente.nl 


\section{$1 \quad$ Begriffsbestimmung}

Constructive Technology Assessment (CTA) ist eine Vorgehensweise für die vorausschauende Gestaltung von Technologie. Das Hauptziel des CTA ist es, laufende Innovationsprozesse zu verbessern, indem ein breites Spektrum wichtiger Aspekte (Akteurinnen, Themen, Realisierungsbedingungen) aufbereitet und mit relevanten Akteure diskutiert werden. CTA setzt daher oft frühzeitig an, solange die Innovation noch gestaltbar ist. Je später, desto schwieriger wird es, neue Erkenntnisse einzubeziehen. Worin die Verbesserung konkret bestehen könnte, gilt es im Zuge der CTA-Interaktion auszuhandeln.

Der Schwerpunkt liegt auf dem inhaltlichen Austausch zwischen Akteurinnen mit verschiedensten relevanten Perspektiven - nicht auf Kreativitätstechniken unter Absehung von Inhalten. Solide Forschung im Vorfeld ist entscheidend für die Qualität der Interaktion mit den Teilnehmenden. Gegenstand von CTA-Interaktionen ist häufig eine Form von Szenarien: hochverdichtete Narrative sowie textlich und grafisch aufbereitete sozio-technische Konfigurationen, Multi-Path-Maps (Innovationspfadkarte) oder Innovationswertschöpfungsketten. CTA-Workshops bieten eine gute Gelegenheit, die koproduktiven Dynamiken (Jasanoff 2004) von Feedback- und Lernprozessen zu nutzen. Weil der Ansatz relativ freie Deliberation und Out-of-the-BoxDenken erfordert, stößt er an Grenzen, wenn Beteiligte aus hierarchisch strukturierten Zusammenhängen kommen bzw. eine CTA unter hierarchischen Bedingungen stattfindet.

Das Constructive Technology Assessment wurde in den Niederlanden entwickelt (Rip et al. 1995) und unter Beteiligung von Forschenden der Universität Twente in verschiedenen Projekten umgesetzt (z. B. Robinson 2010; Te Kulve 2011). Statt auf prinzipielle oder ideologische Kritik zu setzen (sozusagen ,gegen das System“), hat das CTA zum Ziel, einen brauchbaren Beitrag zur jeweils aktuellen Konstruktion neuer Technologien und deren gesellschaftlicher Einbettung zu leisten (,,im/mit dem System“). Beide Ziele werden, je nach Kontext, gemeinsam oder auch getrennt verfolgt. Ich porträtiere CTA hier als Verfahrensansatz, nicht als policy framework (anders: Hack 1995; vgl. auch Bijker 2014 und Rip 2011 zu dem Verhältnis). Als allgemeine frameworks der Reflektion auf Technologieentwicklungen und gesellschaftliche Bezüge wären hingegen Technikfolgenabschätzung im Allgemeinen, Ethical Legal Social Implications (ELSI) und Responsible Research and Innovation (RRI) zu verstehen. Dies sind Meta-Governance-Rahmen, die mit ganz unterschiedlichen spezielleren Verfahren bedient werden. CTA ist gelegentlich eines davon (vgl. Fisher und Rip 2013).

Da CTA mittlerweile auch für andere als technische Innovationsbemühungen verwendet wird, wird im Folgenden oft allgemein von neuen Entwicklungen oder Innovationprozessen die Rede sein. Wo der Ansatz zunehmend als Mittel zur Gestaltung von wissenschaftlichen, produktbezogenen und Governance-bezogenen Neuentwicklungen verwendet wird, zeichnet sich der Gebrauch des Begriffs ,Constructive Innovation Assessment‘ (CINA) ab (Aukes et al. 2020a). Zwei ähnlich orientierte Verfahren sollen kurz Erwähnung finden: das sogenannte ,Real-time Technology Assessment‘ (real-time TA), als Parallelaktion mit natur- und ingenieur- 
wissenschaftlicher Forschung (Guston und Sarewitz 2002), sowie ,Socio-Technical Integration Research ' (STIR), die kommunikative Reflektion über wissenschaftlichingenieurische Routinen im Feld selbst, mit dem Ziel, diese im direkten Arbeitszusammenhang behutsam aufzubrechen (Fisher et al. 2006). Beide liefen zeitweise parallel mit den CTA- und ELSI-Entwicklungen. Auch Real-time TA ist eine der Quellen von STIR.

Der in diesem Beitrag gegenüber der Orientierung auf die Praxis von CTA und CINA weniger beleuchtete theoretische Unterbau kann aus Lothar Hack (1995) und Rip und van den Belt (1986) gut ergänzend rezipiert werden.

\subsection{Die Idee des Constructive Technology Assessment}

Die Kernidee der „konstruktiven“ Einschätzung einer Technologie oder anderer Innovationskandidatinnen besteht darin, dass es mit dem passenden Verfahren möglich ist, zur Gestaltung einer technologischen oder anderer Neuentwicklungen beizutragen. Die Frage, wie man einen Beitrag leistet, ist nicht trivial. Folgt man David Collingridge (1980), so gibt es in einem frühen Stadium der Entwicklung einer technischen Neuerung viel Gestaltungsspielraum, aber vergleichsweise wenig Hinweise darauf, in welche Richtung sich der Gestaltungsspielraum verringert hat, wenn erste Konsolidierungen stattgefunden und neue Routinen sich erst einmal etabliert haben. $\mathrm{Zu}$ einem späteren Zeitpunkt hingegen ist die zukünftige Innovation zwar etwas leichter einzuschätzen, da sich bereits einigermaßen stabile Pfade abzeichnen, der Gestaltungsspielraum dürfte sich jedoch verringert haben, da sich bereits eben einige praktische Routinen und andere Konsolidierungen ergeben haben. Neben diesem WissensKontroll-Dilemma besteht häufig auch ein Antizipationsdilemma: Innovationsträgerinnen möchten warten, bis die Umstände klarer werden, dürfen aber nicht zu lange warten. Was „Zu lange“ bedeutet, ist auch herauszufinden (Rip 2018, S. 56). Bevor sich eine Innovation stabilisiert, sucht man mit $\mathrm{CTA}^{1}$ also die Möglichkeit, auf strukturierte Weise über Innovationsalternativen zu reflektieren. Dazu werden meist sehr explizit die Bedingungen durchgespielt, unter denen eine Innovation, ihre Entwicklung und Förderung realisiert werden könnte.

Handelnde, die in Bezug auf eine Innovation unterschiedliche Rollen und Positionen einnehmen, haben zumeist auch unterschiedliche Perspektiven auf sie und bewerten ihre Chancen und Grenzen unterschiedlich. Man spricht von enactors jenen Handelnden, die eine Innovation aktiv vorantreiben und in den Mittelpunkt ihrer Überlegungen stellen -, und von selectors, für die diese Innovation nur eine Option unter mehreren ist und ihre Auswahl in Erwägung ziehen könnten. Um eine Brücke zwischen diesen beiden Arten von Handelnden und ihren Standpunkten zu schlagen und den Austausch zwischen diesen zu erleichtern, bietet das CTA spe-

\footnotetext{
${ }^{1}$ Wenn im Folgenden von CTA gesprochen wird, ist CINA stets mit gemeint, sofern nicht ausdrücklich anders angegeben.
} 
zielle Gelegenheiten und Anlässe für gemeinsame Überlegungen in einem relativ geschützten Raum (realisiert in der Regel durch einen oder mehrere Workshops).

Weil mit CTA ein Beitrag zur weiteren oder künftigen Entwicklung einer Innovation angestrebt wird, liegt das Hauptaugenmerk auf den möglichen zukünftigen Formen, die eine Entwicklung annehmen könnte im Rahmen dessen, was man bislang über sie weiß - und über die Bedingungen der Möglichkeit. Die Strukturen vorgängiger Entwicklungen werden erfasst, schon um die vorhandenen Entwicklungsmuster begreifen und einkalkulieren zu können. Angesichts der oft hohen Variabilität von Innovationen und der grundsätzlich begrenzten Antizipationsmöglichkeiten (Zukunft ist nach wie vor nicht vorhersehbar), wird vornehmlich zum einen mit Mustern und Regelmäßigkeiten sich schon abzeichnender Entwicklungen gearbeitet, zum anderen werden auch an Hand von Kontextinformationen plausibeldenkbare Ausprägungen in verschiedene Richtungen oder Kombinationen von Aspekten spekulativ eingesetzt. Das Gedankenexperiment ist jedoch nicht rein spekulativ. Empirische und theoretische Erkenntnisse werden ganz umfänglich herangezogen: empirische, indem die gegenwärtig rekonstruierbare Innovationssituation erforscht wird und auch die Perspektiven und Einschätzungen der beteiligten Handelnden; theoretische, indem passende konzeptionelle Angebote verwendet werden, um typische Aspekte systematisch ein Stück weit verallgemeinern zu können.

Dynamiken und Mustern in verschieden stabilen und ausgedehnten Konfigurationen von soziotechnischen Elementen (hinsichtlich Akteursanzahl und Grad der Ausrichtung der Elemente aneinander; vgl. Geels 2011, S. 26, 37-38) wird eine maßgebliche Rolle zugeschrieben: von lokalen Mikroprozessen in „Nischen“ über die Dynamik in bestimmten Bereichen mittlerer Reichweite (relevante Organisationen, Politik- und/oder Arbeitsfelder, Sektoren, usf.) bis hin zu breiteren „Landschaftsentwicklungen“ (also nicht direkt einflussnehmenden, aber die Umstände doch zumindest indirekt prägenden Entwicklungen), die in der begleitenden Forschung oft auch als ,multi-level perspective“ (Geels und Schot 2007; Rip 2012) entfaltet wird. Dynamiken natürlicher/biophysikalischer und technowissenschaftlicher, geschäftlicher und Governance-bezogener Art werden nach Bedarf einbezogen.

Praktisch heißt das oft, dass Analysen des Politikfelds, der Akteurskonstellationen, der Technikentwicklung, des Marktes und der Nutzungsweisen durchgeführt werden. Diese Muster für eine bestimmte Innovation zu kennen und vorwegzunehmen, soll den Handelnden im Innovationszusammenhang helfen, für die Zwecke der Szenariokonstruktion zumindest einige grundlegende Aspekte dessen, was für eine bestimmte Art von Entwicklungs- und Innovationsformat typisch ist, sorgfältig zu antizipieren, soweit dies in Szenarien beschrieben und in einer reflexiven und kontrollierten Spekulation diskursiv geleistet werden kann. Die dadurch angeregte kollektive Spekulation kann, wenn sie kompetent vorbereitet wird, Teil des Innovationsprozesses werden. Hier wird ausgehandelt, was die enablers und selectors tatsächlich zu verfolgen erwägen. Neben der empirischen Fundierung werden mitunter auch Szenarien entlang unterschiedlicher theoretischer Modelle durchgespielt. Theorie wird hier heuristisch eingesetzt. 
Der CTA-Ansatz kombiniert fundierte Forschung zu Innovationssituationen mit einer Reihe von Stakeholder-Workshops und der Anfertigung von verschieden Arten von Szenarien (Narrative, Fiktionen, Roadmaps, Multi-Path-Maps). Die Forschungsergebnisse fließen in die Vorbereitung der Workshops ein und können von interessierten Stakeholdern als ,strategische Intelligenz' (Kuhlmann et al. 1999) für ihre Innovationsprojekte sowie von den Forschern zur wissenschaftlichen Reflexion und Veröffentlichung genutzt werden. Der Ansatz zielt darauf ab, ein fruchtbares Umfeld zu schaffen, in dem die Interessierten die Welten der anderen erforschen können. Am Ende des Tages geht es um tatsächliche Innovationen, nicht nur um ein Spiel oder bloße Spekulation. Wirkliche Akteurinnen mit realen Ideen und Zwängen treten in eine Interaktion ein, die reale Folgen haben können. Ihr Engagement, ihre Ambitionen, ihre Erfahrungen, ihr Ringen, ihre Frustrationen, ihr Konsens und ihre Meinungsverschiedenheiten sind auch real.

\subsection{Praktische Aspekte}

Da der CTA-Ansatz ein praktisches Verständnis der Innovationsbemühungen erfordert, werden diese in ihrem konkreten Kontext und in direkter Kommunikation mit den beteiligten Handelnden erforscht. Im Vorfeld wird eine gründliche Untersuchung zur aktuellen Situation der angestrebten Innovation und der Begleitumstände durchgeführt. Diese kann alle Arten von Methoden nutzen, die für das jeweilige Thema geeignet sind. Dadurch machen sich die CTA-Agenten, die auch die Workshops moderieren, mit den Perspektiven, Interessen, Visionen, Interaktionen und Geschichten der wichtigsten Stakeholder bestens vertraut.

Dadurch können diejenigen, die einen CTA-Workshop vorbereiten und durchführen, die Diskussion durch spezifische Hinweise anregen und relevante Themen und Stakeholder mit einem Gespür für die Situation einbeziehen. Man muss also einschätzen können, welche Art von Themen und Beiträgen diese in der Regel leisten. Dies ist wichtig sowohl bei der Komposition des Workshops (Einladung, Festlegen des Rahmens, der Thematik, der Interaktionsformate) als auch während der Durchführung des Workshops selbst. Gut informierte, realistische und zum Nachdenken anregende Szenarien (Erzählungen, konzeptuelle Grafiken, symbolische Bilder usw.) sind Kernwerkzeuge. Die Vorbereitung kristallisiert sich oft noch präziser in der Entwicklung von Szenarien heraus. Externe Moderation ist schwierig, weil diese meist nicht aus dem vollen Kontextwissen schöpfen kann.

Die Workshops werden als sichere Räume (protected spaces; Rip und van den Belt 1986) moderiert, in denen diejenigen, die sich der kollektiven Reflexion einer Innovation zuwenden, Erwägungen und Alternativen jenseits der üblicherweise von ihnen gewählten Blickwinkel in Betracht ziehen können (oder dazu herausgefordert werden): ,probe each other's ,realities““ (Garud und Ahlstrom 1997, S. 44) genannt. Die Workshops dienen als Anlässe für die Formulierung von Strategien, Bedarfen und so fort mit dem Ziel: 
- die Beteiligten als gut informierte Experten für ihre eigene Situation ernst zu nehmen;

- die Innovationskontexte als echte Aushandlungszusammenhänge zu verstehen, in denen es um reale Dinge geht für die Beteiligten;

- gegenseitiges Lernen zu ermöglichen über die Möglichkeiten und Grenzen einer Innovation, die Perspektiven anderer Akteurinnen, wie weit man sich unterhalten oder sogar mit anderen zusammenarbeiten kann usw.;

- die Begegnung mit Akteuren herbeizuführen, die an Ähnlichem interessiert sind, was konstruktive Inspiration bieten kann, auch und gerade wenn sie ihre eigenen Vorstellungen verfolgen oder die Dinge anders sehen (denn dadurch werden alle Beteiligten dazu gezwungen, sich auch mit Fragen jenseits komfortablen Konsenses auseinanderzusetzen);

- weitere Vernetzung (falls nicht nur ein einmaliger Austausch bezweckt ist) zu begünstigen, wobei die strategischen Ziele und Überlegungen der Akteurinnen explizit dargelegt werden und Möglichkeiten der Zusammenarbeit bereits vorab und natürlich während der Workshops ausgelotet werden müssen (es kann etwa darum gehen, eine Innovationsplattform aufzubauen, an der die Teilnehmenden weiter mitwirken).

Die CTA-Interaktionen werden selbst mit empirischen Methoden dokumentiert und analysiert, um systematisch reflektierte Ergebnisse produzieren zu können.

Die Workshops müssen spezifisch sein in Bezug auf die vorliegenden Fälle, die beteiligten Akteure und die in den Szenarien diskutierten Optionen; auch offen genug und Denkgrenzen sprengend, um zu kritischen Bemerkungen, Gegenargumenten und Alternativen einzuladen:

- mit expliziten Angaben zu Optionen: unterstützt durch (sozio-ökologische, Governance-bezogene, physische) Szenarien, in denen mögliche Konstellationen, die für eine Innovation fruchtbar sind, klar zum Ausdruck gebracht werden, einschließlich ambivalenter, unbekannter, nicht erreichbarer oder nicht kontrollierbarer Situationen;

- indem konstitutive Elemente klar definiert werden: Optionen sind erst dann sinnvoll, wenn Aspekte erläutert werden, auf denen die Szenarienoptionen basieren;

- indem sie insbesondere zukunftsspezifisch sind: Szenarien, die die möglichen kurzfristigen (oder auch mittelfristigen) Zukünfte widerspiegeln, indem bestehende Trends extrapoliert und die Bedingungen für Veränderungen so genau wie möglich herausgearbeitet werden;

- indem sie Bedingungen der Möglichkeit, dass eine Option tatsächlich funktioniert, explizieren: um besser darüber reden und nachdenken zu können, was zu einer Zukunft für die betreffende Innovation führen könnte. 
Die daraus abgeleiteten Szenarien sollten sinnvollerweise:

- in die Abschätzung der potenziellen Auswirkungen von Aktivitäten und in die Entwicklung von Strategien einfließen, wobei die gewünschten Ergebnisse und unbeabsichtigten Auswirkungen berücksichtigt werden;

- einen individuellen und kollektiven Denkraum eröffnen, um gemeinsam mit den Hauptakteurinnen entscheidende Probleme für die Optionen und Wege zu identifizieren, sowohl in Bezug auf potenzielle Probleme als auch in Bezug auf Machbarkeit und Nutzen;

- sowohl diejenigen einschließen, die die Innovation in die Tat umsetzen (weil sie es für sinnvoll halten; die enactors), als auch diejenigen, die sie möglicherweise auswählen (sobald sie die innovativen Ergebnisse interessant genug finden, nützlich, wünschenswert oder aus einem anderen Grund auswählen; die selectors); dazu kann es nötig sein, auch Dritte einzubeziehen, die noch nicht direkt involviert sind, aber als potenzielle Partner vielversprechend sind, um herauszufinden, wie bestimmte politische oder Marktbedingungen, Geschäftsmodelle und technologische oder wissenschaftliche Aspekte oder Bedingungen einer Innovation realisierbar werden können;

- den Stakeholdern zeigen, wie andere Akteurinnen, die normalerweise nicht beteiligt sind, tatsächlich für die Weiterentwicklung der Innovation entscheidend sein können;

- so verfasst sein, dass sie als Entscheidungshilfe für die Auswahl von Teilnehmern verwendet werden können, die tatsächlich eingeladen werden müssen, um das gesamte Spektrum relevanter Perspektiven am Tisch zu haben und gehört zu werden;

- so verfasst sein, dass sie die Erkenntnisse über die Innovationssituation und Akteursperspektiven/-interessen aus der vorgängigen Forschung stark verdichten und in einen kohärenten Zusammenhang bringen, was regelmäßig am besten gelingt, wenn ein textliches Narrativ verfasst wird, für welches die ausgewählten Aspekte und Zusammenhänge explizit ausformuliert und erläutert werden in einer Sprache, welche die beteiligten Akteure verstehen.

Die Szenarien spiegeln - wenn sie gut gemacht sind - realistisch die Situation wider, in der sich die Teilnehmenden befinden oder die sie anstreben. Die Perspektiven der verschiedenen Akteure sollten sichtbar werden ebenso wie die Spannungen und Dilemmata, die sich daraus ergeben könnten.

Realistisch sein bedeutet nicht, nur vorsichtige oder moderate Entwürfe zur Diskussion zu stellen. Extremere Alternativen (die also hoch selektiv und pointierte Wege aufzeigen oder radikal zum Umdenken nötigende) können sehr stimulierend wirken, um Möglichkeiten und Grenzen der Machbarkeit und Wünschbarkeit auszuloten. Sie werden oft jedoch umso spruchreifer, je mehr gezeigt werden kann, unter welchen Bedingungen sie sogar Aussicht auf Realisierung bergen - trotz ihrer Radikalität oder Selektivität. Letztlich ist es eine Frage der Kunst der Moderierenden bei der Handhabung der Szenarien während der Interaktion zu merken, wie weit man die Provokation treiben kann, um anregend zu sein, und wie machbar etwas sein muss, um auch wenigstens in die Nähe von Umsetzungsmöglichkeiten zu gelangen. 
Das Format der Vorlagen für die Workshopdiskussion kann je nach Zielsetzung und Entwicklungsstand der Innovation variieren:

a. Wir haben zunächst von hochverdichteten, bestens informierten textlich-narrativen Szenarien (Rip und Te Kulve 2008; Parandian 2012) als Grundform gesprochen. Man muss sie sich vorstellen wie Kurzbeschreibungen zukünftiger sozialer Welten, welche vor allem sozio-technische, politische, moralische, ökonomische, ökologische Veränderungen umreißen sowie inhärente Dilemmata; wenn man so will, wie fiktionale Kurzgeschichten. Die Kunst ist, zugleich ausgeprägten Realismus und konstruktive Denkprovokation zu leisten.

b. Daneben verwendet man sozio-technische Konfigurationen (Geels 2006; Hulshof 2016), die sowohl textlich als auch grafisch aufbereitet werden. Diese entfalten expliziter als die narrativen Szenarien, in welche sozialen Gefüge Technologien eingebunden sind. Sie berücksichtigen die direkten Gebrauchszusammenhänge (die Artefakte, Nutzerinnen und deren Gebrauchsweisen nebst Infrastrukturen). Sie enthalten zusätzlich einen Blick auf den Lebenszyklus (Herstellung und Bereitstellung neben Vertrieb und Wartung sowie Entsorgung und Recycling) und reflektieren darüber hinaus, wie sich die Innovation in die soziale Ordnung einfügen lässt (darauf bezogene Politikgestaltung und Regulierung ebenso wie kulturelle und symbolische Bedeutungsmuster). Dabei kann man eine aktuell vorliegende Konfiguration besprechen, etwa in Analogie zu konventionellen Produkten, die bereits auf dem Markt sind. Man kann Alternativen entwickeln, welche die Innovation etwa für verschiedene Nutzungskontexte, Jurisdiktionen oder Märkte entfaltet. So kann herausgearbeitet werden, wo die kritischsten Aufgaben lauern und sich unerwartete Übereinstimmungen zwischen unterschiedlichen Stakeholdern ergeben.

c. Mehrpfadkarten (Robinson und Propp 2008; van Lente und van Til 2007) kommen zum Einsatz, wenn man sich im frühen Entwicklungsstadium einer Innovation befindet, und noch recht offen ist, welche späteren Anwendungskontexte sich wie etablieren werden. Der Vergleich mehrerer denkbarer Pfade erlaubt es, mehrere alternative Anwendungen, Governanceregime oder Marktverhältnisse durchzuspielen. Es geht sowohl um eine strukturierte Voreinschätzung von Politik-, Regulierungs-, Produkt-, Markt- und Verwendungsalternativen als auch darum, mit denen Teilnehmenden eventuelle Partner für die weitere Entwicklung und Vermarktung zu finden. Die Vorgehensweise ist angelehnt an das Managementwerkzeug des Roadmapping, benutzt aber Heuristiken aus der Innovationsforschung, wie die ,transition pathway typology ' der Multi-Level-Perspektive (Geels und Schot 2007), wenn die rein technische, betriebswirtschaftliche oder marketingbezogene Sicht zu eng erscheint. Die Entwicklungspfade können sowohl im Workshop gemeinsam erarbeitet werden als auch vorab erstellt worden sein und im Workshop für ein Backcasting verwendet werden.

d. Innovationen laufen nicht linear ab und halten sich nicht an theoretisch modellierte Schrittfolgen (Kline und Rosenberg 1986; Deuten et al. 1997; Gümüscü 2016). Innovationswertschöpfungsketten werden dennoch in den Blick genommen, um sich über verschiedene Aspekte eines Innovationsablaufs generell klarer 
$\mathrm{zu}$ werden. Während die zuvor angesprochenen Mehrpfadanalyen verwendet werden, um sich abzeichnende Entwicklungsalternativen konkret durchzudeklinieren, dienen die Analysen mit Hilfe von Innovationswertschöpfungsketten der Einschätzung, welche grundsätzlichen Herausforderungen auftauchen könnten, wenn man Aspekte wie etwa Forschung, Fertigung, Industrie- und/oder Marktgängigkeit, Regulierung, Politikgestaltung, allgemeingesellschaftliche Aufnahme einer Innovation mitbedenkt. Nicht der Pfad, sondern die Elemente einer wie auch immer sich entwickelnden Kette stehen im Mittelpunkt und werden eher pauschal ausgelotet.

e. Daneben finden auch ganz basale Analyseweisen Verwendung, etwa zu Konstellationen und Dynamiken von Akteuren, Technologien bzw. soziotechnischen Systemen oder zur Governance bzw. zum Politikfeld. Hier geht es um sehr begrenzte, speziell fokussierte Betrachtungen, die zum Einsatz kommen, wenn noch zu wenig über die Innovation selbst bekannt ist oder wenn Bedarf besteht eine bestimmte Dimension gesondert anzuschauen.

Überall hier dienen die Szenarien und anderen Formate sowohl zur Teilnehmendenauswahl in der Vorbereitung als auch als Gesprächsgrundlage für den Workshop und als wertvolle Dokumentation von strategischen Erkenntnissen zum Mitnehmen und Weiternutzen. Gute Szenarien werden von den Teilnehmenden als dermaßen nützlich erachtet, dass sie diese mit „,nach Hause“ nehmen und weiterverwenden.

Die Rolle der Organisatoren und Moderatorinnen ist es, sich über die Gegenstandsbereiche, die für die jeweilige CTA relevant sind, kundig zu machen (vgl. Rip 2018, S. 1): sich in den Bereich, in dem sich die Akteurinnen, die am CTA teilnehmen, einzufügen und dort neugierig umzusehen; verschiedenste Teilbereiche und Akteurinnen zu erkunden; und Unterschiede und Verbindungen zu suchen, die neue Impulse geben. Nicht alle Teilnehmerinnen werden dies selbst in gleicher Weise können (aus Ressourcenmangel weil sie ein eingefahrenen Denkweisen gefangen sind). Die Aufgabe von CTA hingegen ist es, Fragen zu stellen und Alternativen anzusprechen, die in der Form unter den Akteuren zwar nicht üblich, sachlich aber auch nicht abwegig sind. Es geht darum, „um die Ecke zu denken“ und eine Vermittlerrolle zu spielen. Einerseits nimmt man damit eine große Verantwortung auf sich. Wie handlungsentlastend könnte es hingegen sein, eine herausgehobene oder desinteressierte Position einzunehmen und sich nicht um die Erfahrungsebene der Akteure zu kümmern. Zugleich ist dies aber auch eine sehr bescheidene Rolle, die eben nicht immer schon weiß, was richtig oder wie etwas zu deuten ist - vielmehr eine Rolle, die zwischen Akteurinnen und Sachbefunden moderiert.

\subsection{Der Innovationsbegriff des CTA}

Die Innovationsperspektive des CTA und des CINA-Ansatzes beinhaltet eine Vorstellung von Innovationsdynamiken mit drei Kernherausforderungen für diejenigen, die sich an einen Innovationsprozess beteiligen wollen: (1) laufende Dynamiken von technologischen Innovationen $\mathrm{zu}$ verstehen; (2) zu versuchen, sie zu modulieren; 
und (3) Akteurinnen wechselseitige Lernprozesse zu ermöglichen und sich als „CTA-Bewerkstelligende“ selbst davon nicht auszunehmen, sondern sich der eigenen Rolle auch selbst-reflexiv zuzuwenden (Rip 2018, S. 52). Vertreterinnen von CTA und CINA betrachten Innovationen als hochgradig dynamisch ablaufende Entwicklungen, die am ehesten mit einer evolutionären, nicht linearen Perspektive greifbar sind: gekennzeichnet durch Multi-Akteur-Interaktionen, Prozesse verteilter Initiative sowie Kontingenzen, die zwischen den Polen Stabilisierung und Destabilisierung bzw. Ausrichtung (alignment), Diffuswerden (dealignment) und Neuausrichtung (realignment) oszillieren.

Innovationen finden nicht im luftleeren Raum statt - auch wenn diejenigen, die an ihnen arbeiten, mitunter so tun (müssen), als gehe es um nichts anderes, als die Generierung und Reifung des Neuen und Besonderen, mit dem sie gerade beschäftigt sind. Früher oder später muss es aber gelingen, das Neuartige in eine existierende Ordnung einzufügen oder die existierende Ordnung entsprechend zu ändern (vgl. Rip 2018, S. 52). Im Erfolgsfall kommt es zu der Domestizierung einer neuen Technologie, im Zuge derer sie in lokale Alltagspraktiken eingebettet und mithin von Nutzerinnen angeeignet (appropriation) wird. Zugleich hat man beobachten können, dass der Weg von den Anfängen über Erwartungen und Versprechungen bis zur gelungen Einpassung in die bestehende soziale Ordnung nicht geradlinig verläuft und zudem mit Hindernissen gepflastert ist. Nicht alle relevanten Akteure und Systeme sind schnell bereit, das Neue anzunehmen, sondern warten zunächst ab (,waiting games ${ }^{\mathrm{s}}$ ), bis auch andere sich rühren (Parandian et al. 2012).

Zwar entsteht eine Innovation vor dem Hintergrund bestehender soziotechnischer oder anderer Regime. Sie kann diese mit der Zeit jedoch auch verändern. Der Begriff des „bestehenden Regimes“ (incumbent regime) bezieht sich auf eine Reihe von Regeln, Praktiken und Institutionen, die die Weiterentwicklung einer Technologie (Dienstleistung, Markt, Politik) strukturieren (Geels und Schot 2007). Das bislang dominante Design einer Technologie, das Arrangement eines sozio-technischen Regimes oder eine Governanceinnovation kann durch sich durchsetzende Neuerungen innerhalb eines dominanten Designrahmens zur Veränderung gedrängt - oder in Teilen oder ganz geschwächt, unterminiert, verdrängt werden (Geels und Schot 2007; Turnheim und Geels 2012; Stegmaier et al. 2014). Solche Variation oder Abkehr kann auch durch Antizipation ausgelöst werden: wenn man nicht wartet, bis alle anderen sich auch verändern, sondern wenn man sich an die Spitze der Erneuerung setzt in Erwartung etwa eines strategischen Vorteils. Die Veränderung kann sich gerade deshalb zu neuen Regimearrangements oder gar Paradigmen stabilisieren, weil man den Umbau offensiv angeht und andere zum Mitziehen gewissermaßen zwingt (Rip 2018, S. 53; Dosi 1982). Diese Sichtweise eröffnet einigen Spielraum, um im Rahmen einer CTA strategische Alternativen durchzuprobieren.

Das CTA versteht sich als ein Mittel, um relevant erscheinende Kontextentwicklungen zu identifizieren und durchzuspielen. Der Herausforderung der Antizipation in einer ungewissen und weitgehend unvorhersagbaren Welt wird oft dadurch begegnet, dass man Komplexitätsreduktion betreibt, indem man auf geteilte Erwartungen setzt. Diese Erwartungen werden durch ein CTA sowohl mit Blick auf das im 
Fokus befindliche Regime als auch hinsichtlich verschiedener als relevant eingeschätzter Kontextdynamiken konkretisiert. Im Umgang mit Ungewissheit sind innovierende Akteurinnen wie auch CTA-Agenten oft darum bemüht, einige Varianz aufrechtzuerhalten und die Innovationen im Werden vor zu harter Selektion zu beschützen. Die oben kurz erwähnten „Nischen“ haben diese Funktion. CTA-Szenarien sollen jedoch auch die die Grenzen der Variabilität und der Schutzbedürftigkeit ausloten.

Stakeholder sind bestrebt, eine neue Entwicklung aus Sicht ihrer Ziele zu beeinflussen (Kuhlmann et al. 2019). Einen solchen Einfluss üben auch die Assessments, die Handlungen (oft versuchsweise nächste Schritte) und Interaktionen dieser Akteure aus (Rip 2018, S. 55-56). Hier setzen CTAs an und bieten einen Rahmen für strukturierte Interaktion und systematische Aufbereitung der Ausgangslage - sie kanalisieren und moderieren idealerweise die bestehenden Versuche der Innovationsbeeinflussung. Zugleich verändert die Intervention die Entwicklung und muss daher stets aufs Neue vermessen werden. Im Innovationsprozess selbst finden mehr oder weniger reflexive Lernprozesse (Lernen erster und zweiter Ordnung) statt, und das CTA reklamiert, diese auf besonderes konstruktive Weise zu unterstützen.

Die CTA-Aktion wird als Teil von Innovationsprozessen angesehen, die durch explizite Bemühungen um Reflexion begleitet werden (vgl. Van de Poel 2000 zur der Rolle von Außenseitern und Alternativprodukten für die Veränderung soziotechnischer Regime). In ihrer Wirkungsweise als Nexus zwischen den Variations- und Selektionszusammenhängen in der Ko-Evolution von Technik und Gesellschaft wird sie in einem Atemzug mit ,bridging events ${ }^{6}$ zur Vermittlung zwischen Insidern und Outsidern genannt (Garud und Ahlstrom 1997) und als convergence work (Stegmaier 2009) konzipiert.

\section{Stand der Forschung - ausgewählte Studien}

\subsection{Forschung mit Hilfe von CTA}

Man kann die Forschung, die mit Hilfe von CTAs durchgeführt wird, mit Blick auf die intrinsische oder extrinsische Zielsetzung ihrer Verwendung unterscheiden. Im ersten Fall soll den direkt an einer Innovation Beteiligten die Gelegenheit zur Betrachtung ihres Gegenstands und ihrer Zielsetzungen im (weiteren) sozialen Kontext gegeben werden (von der Innovation zum Kontext). Im zweiten Fall dient die CTA-Aktion auch der Erfassung von und Auseinandersetzung mit Unsicherheiten und anderen Grenzen der Wünschbarkeit jener Richtung(en), die eine Innovation nehmen kann. Hier geht es darum, aus der Perspektive der breiteren gesellschaftlichen Bedeutung einer Innovation auf die Entwicklungsbemühungen zu blicken (vom Kontext zur Innovation; vgl. van Merkerk und Robinson (2006); Rip und Te Kulve (2008)).

Mit Bezug auf die selbst gesteckten und unmittelbaren Forschungsziele lassen sich Strategieartikulation (strategy articulation; vgl. Robinson 2010; Te Kulve 
2014) und Bedarfsartikulation (demand articulation; Te Kulve und Konrad 2017) unterscheiden.

\subsection{Forschung und Reflexion über CTA}

Es liegt eine ganze Reihe von Reflektionen und Analysen vor, in denen sich die Proponenten von CTAs der eigenen Arbeit kritisch zuwenden (zu CINA sind diese erst im Entstehen). Der Ansatz speist sich auch aus der Tradition und aus Einsichten empirischer Science and Technology Studies (STS), in denen die Analyse der Bedingungen des eigenen Forschens und der Rolle der eigenen Forschung im weiteren politischen und gesellschaftlichen Kontext üblich ist (Rip 2002; Te Kulve 2011; vgl. auch Marris et al. 2008; Wynne 1975).

\section{$3 \quad$ Grenzen des Ansatzes}

Kritikpunkte am CTA (CINA ist zu jung) sind, dass Machtstrukturen theoretisch zu wenig adressiert werden, wobei aber konzediert wird, dass dieser Fokus in Empirie und Ausführung zum Tragen kommt (Hack 1995). Daneben ist die Rede von mangelnder Unterscheidbarkeit von Produktentwicklung und Vernachlässigung der Frühwarnfunktion der Technikfolgenabschätzung (Smits 1992, S. 265-266). Kritisiert wird auch eine Vernachlässigung der Besonderheiten und Unterschiede von Technologien (Vergragt und Groenewegen 1989). Außerdem wird mangelnde Neutralität und Nutzbarkeit von CTA als Ressource der (politischen) Gestaltung von Technologie kritisiert (Vergragt und Groenewegen 1989, S. 30). Laut Bijker (2014, S. 28) könne das CTA zudem schlecht mit jenen Aspekten umgehen, die nicht in Szenarien gefasst werden können.

CTA und CINA funktionieren nicht wie beschrieben, wenn (a) die im Fokus befindliche Situation nicht gründlich erforscht ist, (b) nicht ausreichend Zeit und Engagement zur Auseinandersetzung mit den Szenarien besteht (Bijker 2014, S. 28) oder (c) exakte Vorhersagen erwartet werden. Letztendlich steht und fällt der Ansatz mit der Einsicht, dass er eher eine Umgangsweise mit Ungewissheiten und Optionen bietet als eine konkrete Lösung. Am meisten kann erreicht werden, wenn CTA und CINA in einen weiteren Innovationsprozess eingebaut werden, statt mit einmaligen Workshops. Diskussionen über die Durchführbarkeit von CTAs in Ländern mit nicht-freiheitlichen Regierungsformen legen überdies nahe, dass die umfassende Geltung von Freiheitsrechten unabdingbar ist, um offen und sachorientiert, statt lediglich nach Maßgabe einer Obrigkeit oder einer anderen Autorität sprechen zu können. Leonhard Hennen und Linda Nierling (2014) weisen auch auf die Notwendigkeit der passenden civic epistemology (Jasanoff 2005) hin, also der institutionellen Praktiken der Wissensgenerierung und -verwendung bei der Politikberatung, Politikgestaltung und Entscheidungsfindung, was man auch eine postpositivistische Haltung (Héretier 1993) nennen könnte. 


\section{$4 \quad$ Ausblick: Weiterentwicklungen}

Zwei jüngere Variationen des CTA-Kernansatzes sollen abschließend beleuchtet werden. Zum einen haben sich ,abgespeckte“ Formate im Kontext von Qualifikationsprojekten und angewandter Forschung herausgebildet. Zum anderen geht es um die Übertragung des CTA-Gedankens auf nicht-technische oder nicht primär technische Entwicklungen.

\subsection{CTA Lite}

Als „CTA Lite“ firmieren in jüngerer Zeit zwei Versuche, den recht voraussetzungsvollen CTA-Ansatz auf kleinere Anwendungszusammenhänge zuzuschneiden (siehe Tab. 1). Zum Beispiel organisierte eine in CTA angelernte Biologin, nicht eine Sozialwissenschaftlerin, einen CTA-Lite-Workshop mit mehreren Akteurinnen aus verschiedenen Fachgebieten, um mögliche Anwendungen für die mikrofluidische Lipiddoppelschichtplattform zu untersuchen (Schulze Greiving et al. 2016). Das CTA fand hier als Teil eines laufenden Projekts statt, das auf Technologieentwicklung fokussiert war. Entsprechend wurden Elements des „vollen“ CTA-Ansatzes herausgegriffen und den Umständen angepasst. In den Hintergrund getreten ist der Anspruch, sich auf sanfte Weise in einem frühen Entwicklungsstadium einzubringen. Beibehalten wurde das kollektive Mapping und die Szenario-Erarbeitung. Der Workshop wurde nicht zum Bridging-Event, sondern als „Pre-Screening“-Tool potenzieller Anwendungen nanomedizinischer Technologie, die von der Doktorandin aktuell entwickelt wurden und deren Diskussion von den unterschiedlichen Hintergründen der Teilnehmer profitierte. Interviews mit Teilnehmenden zur Vorklärung fanden nicht statt.

Während Verena Schulze Greiving alles in das eine Meeting verlegt und hoch konzentriert die Denkansätze und die weiterführenden Möglichkeiten auslotet, schlagen Hamaker-Taylor et al. (2018) vor, einen CTA-ähnlichen Ansatz durch Interviews mit einzelnen Akteuren, statt in einer Gruppe mit verschiedenen Akteuren umzusetzen. Weil sich die Interessensvertreter im Finanzbereich ungern offen über ihre Geschäftsstrategien auslassen, wurde ein bilaterales Verfahren nötig, das den Gesprächswilligen einen ganz besonders geschützten Raum bot: einen persönlichen geschützten Raum.

Aus ausführlichen Analysen im Vorfeld waren bereits für das Gesamtprojekt hinreichend schlüssige Typen von Serviceinteraktionen im Bereich von meteorologischen klimabezogenen Beratungsdienstleistungen (,climate services“) entwickelt und mit verschiedenen Gruppen von Handelnden durchprobiert worden. Ziel war es gewesen, grundlegende Konstellationen zu finden, die für mögliche Nutzer praktisch so relevant sind, dass man an ihnen besprechen konnte, welche Marktpotenziale für diese neuartigen Dienstleistungen vorhanden sind. Für den Finanzsektor wurden diese Typen noch mit passenden Beispielen angereichert und zur Diskussion gestellt. Die angesprochenen Finanzvertreter gaben nun im Interviewgespräch ihre Einschätzungen, wie in ihrer Firma klimatologisches Wissen Verwendung finden und eine 
Tab. 1 Vergleich von CTA Lite in Gruppen- und Individualvarianten

\begin{tabular}{|c|c|}
\hline CTA Lite (Gruppenvariante) & CTA Lite (Individualvariante) \\
\hline Vorstudien sehr begrenzt & Vorstudien ausführlich \\
\hline Themen und Probleme identifizieren & Typologie als stark zugespitzte Szenarien \\
\hline Auswahl der Teilnehmenden & Auswahl der Teilnehmenden \\
\hline Kollektiver Workshop & Individuelle Gespräche \\
\hline $\begin{array}{l}\text { Mapping mit Teilnehmenden als Ad } \\
\text { hoc-Szenarien-Bildung }\end{array}$ & $\begin{array}{l}\text { Durchbesprechen der Typen als Sondierung der } \\
\text { Kurz-Szenarien }\end{array}$ \\
\hline $\begin{array}{l}\text { Vergleichende Analyse des } \\
\text { Gruppengesprächs }\end{array}$ & Vergleichende Analyse der Einzelgespräche \\
\hline $\begin{array}{l}\text { Hinweise auf zukünftige Verwendungs- } \\
\text { und Entwicklungspotenziale als Ertrag }\end{array}$ & $\begin{array}{l}\text { Hinweise auf zukünftige Verwendungs- und } \\
\text { Entwicklungspotenziale als Ertrag }\end{array}$ \\
\hline $\begin{array}{l}\text { Konkretisierung des Ertrags: } \\
\text { Innovationspfadkarten und -analysen }\end{array}$ & $\begin{array}{l}\text { Konkretisierung des Ertrags: Produktmatrizen für } \\
\text { Dienstleistungen aus Sicht unterschiedlicher } \\
\text { Akteurstypen }\end{array}$ \\
\hline
\end{tabular}

Servicebeziehung etabliert werden könnte. Das Besondere ist hier der Umstand, dass statt ausführlicher Szenarien, lediglich knapp gefasste Typen von Servicemodellen besprochen wurden.

Die CTA Lite-Variante bestand hier darin, zwar Szenarien in konziser Form zu nutzen, statt eines breiteren Perspektivenspektrums, aber nur einzelne Akteure und Akteurinnen auf deren Sicht zu den Servicemodellen anzusprechen. Der Nachteil eines solchen Vorgehens ist, dass man die Sichtweisen von Akteurinnen nicht direkt in Dialog zueinander bringen und beobachten kann. Der Vorteil ist, dass man dennoch ein Objekt gemeinsamen Fokussierens zur Hand hat und wenigstens im Nachgang zu den einzelnen Gesprächen vergleichende Analysen anstellen kann, die in einer angemessenen Form auch an die einzelnen Gesprächspartner zurückgemeldet werden können.

\subsection{CINA: Anwendung des CTA-Ansatzes auf (primär) nicht- technische Innovationen}

CTA wird aktuell vielseitig in der Forschung eingesetzt. In einem Projekt zu ServiceInnovation bei Klimadienstleistungen wurde in Workshops und Interviews eine durch das CTA inspirierte Exploration des Nexus von Bereitstellung und Gebrauch, Organisation und Informationsinfrastruktur sowie technologischen und wissenschaftlichen Aspekten vorgenommen. Der Blick auf sozio-technischer Zusammenhänge ist dabei auf sehr konkrete Weise zugespitzt worden. Da für alle Beteiligten der Gedanke an Technologie und TA fernlag, wurde der CTA-Begriff weitgehend vermieden und eher die Vorgehensweise hervorgehoben.

Der CTA-Ansatz diente als Quelle für Analysen und zur Aufbereitung der Analyseergebnisse: (a) Zunächst ging es um die Analyse von Serviceinnovationen, die zum Ziel hat, ein realistisches Spektrum Relationen zwischen Gebrauch und Erstellung von Dienstleistung zu erarbeiten. Die Dienstleistungen sind einerseits durch 
Bezüge zu hoch entwickelter Technologie und Wissenschaft gekennzeichnet und anderseits durch spezifische Verwendungskontexte und -bedingungen in konkreten Arten von Organisationen in verschiedenen Handlungsbereichen (Finanzdienstleistungen, Tourismus, Stadtplanung). (b) Diese komplexen Verhältnisse mussten greifbar gemacht werden, Optionen für gelingende und Risiken für das Scheitern von Serviceinnovationen mit Expertinnen und Interessenvertreter (und davon jeweils sowohl enablers als auch selectors) besprechbar zu machen. Statt längerer textlich-narrativer Szenarien erschien die Entwicklung kurzer, prägnanter Beschreibungen von Typen von Klimadienstleistungen praktikabler. Zugleich wurde die anfangs noch recht allgemein gehaltene Typologie im Zuge der Besprechung in Workshops weiter korrigiert oder bestätigt, mithin also nach und nach angepasst und verfeinert (vgl. Visscher et al. 2020; Damm et al. 2019).

Erst in einem weiteren Projekt, das auch keinen sehr direkten Bezug zu einer spezifischen Technologie aufwies, sondern im Bereich von Waldökosystemdienstleistungen Innovationen von Governance-Arrangements und Geschäftsmodellen zum Gegenstand hatte, wurde der Ansatz neu etikettiert als Constructive Innovation Assessment (kurz: CINA; Aukes et al. 2020a, b, c). Hier geht es um sozio-technische-ökologische Systeme und die damit verbundenen Formen von Governance und Geschäftsmodellen. In sieben Regionen der EU wurden besonders innovative oder innovationsbedürftige Ansätze, Wald ökologisch und ökonomisch nachhaltig zu bewirtschaften, ausgewählt. Der CINA-Ansatz diente dazu, Foren vorzubereiten und in Form von Workshops anzubieten, in denen die lokal Interessierten und die Projektmitarbeitenden an konkreten Szenarien arbeiten konnten. Entlang der Szenarien konnten die bestehenden Leistungen weiterentwickelt oder neue entwickelt werden. CINA war damit nicht fokussiert alleine auf die Ausrichtung von Workshops, sondern lieferte einen guten Teil an Strukturierung der gesamten Innovationsarbeit: die gründliche Analyse der Ausgangssituation und Vorgeschichte, der Akteurinnen und von deren Perspektiven und Interessen, die Aufnahme von zunächst explorativen, dann je nach Mitwirkungsbereitschaft kontinuierlicher werdenden Konversationen und schließlich auch die laufende Aushandlung der Innovation. Der Innovationsprozess sah drei Arten von CINA-Workshops vor: zur Innovationsanalyse und -vision, zum frühen Assessment des Innovationsprototyps und zum Roadmapping über die Projektlaufzeit hinaus.

\subsection{Didaktisierung und CTA in der Lehre}

Bemühungen der Didaktisierung und Verwendung der Ansätze in der universitären Lehre, zum Beispiel für Lehrforschungs-Projekte, gehen einher mit der Entwicklung der Ansätze selbst. Dort, wo sie entstanden und im Entstehen sind, sind Forschung und Lehre stark miteinander verknüpft: an technischen Universitäten, die natur- und technikwissenschaftliche mit sozial- und geisteswissenschaftlichen Blickwinkeln konfrontieren wollen, die Spielräume und Programme bieten, um etwa Ingenieurinnen sozialwissenschaftlich, betriebswirtschaftlich und philosophisch-ethisch zu sensibilisieren, aufzuklären und zu unterweisen. So ist als Rollenspiel-Simulation 
jüngst das Theatrical Technology Assessment (TTA) entstanden (Visscher 2020). Ziel ist es, die komplexen Wechselbeziehungen zwischen Technik und Gesellschaft spielerisch näher kennen- und verstehen zu lernen und dabei als Studierende des Ingenieurwesens oder der Sozialwissenschaften spielerisch Rollen und Positionen einnehmen, die in der Perspektive ihrer jeweiligen Disziplinen normalerweise unterbelichtet bleiben.

\section{Literatur}

Aukes, Ewert, Peter Stegmaier, und Christian Schleyer. 2020a. Ecosystem Services Governance Navigator \& Manual for its Use. InnoForESt Deliverable 5.5. https://innoforest.eu/repository/ d5-5/. Zugegriffen am 04.01.2021.

Aukes, Ewert, Peter Stegmaier, und Christian Schleyer. 2020b. Final report on CINA workshops for ecosystem service governance innovations: Lessons learned. InnoForESt Deliverable 5.3. https://innoforest.eu/repository/d5-3/. Zugegriffen am 04.01.2021.

Aukes, Ewert, Peter Stegmaier, und Christian Schleyer. 2020c. Set of reports on CINA workshop findings in case study regions, compiled for ongoing co-design and knowledge exchange. InnoForESt Deliverable 4.2. https://innoforest.eu/repository/d4-2-overview/. Zugegriffen am 04.01.2021.

Bijker, Wiebe. 2014. Technology assessment: The state of play. Towards hybrid and pluriform process of governance of science and technology. In Technology assessment and policy areas of great transitions. Proceedings from the PACITA 2013 conference in Prague, Hrsg. Tomas Michalek, Lenka Hebakova, Leonhard Hennen, Constanze Scherz, Linda Lierling und Julia Hahn, 23-36. Prag: Technology Centre ASCR.

Collingridge, David. 1980. The social control of technology. London: Pinter.

Damm, Andrea, Judith Köberl, Peter Stegmaier, Elisa Alonso Jiménez, und Atte Harjanne. 2019. The market for climate services in the tourism sector - An analysis of Austrian stakeholders' perceptions. Climate Services 17:100094.

Deuten, J. Jasper, Arie Rip, und Jaap Jelsma. 1997. Societal embedding and product creation management. Technology Analysis \& Strategic Management 9(2): 131-148.

Dosi, Giovanni. 1982. Technological paradigms and technological trajectories. A suggested interpretation of the determinants and directions of technical change. Research Policy 11:147-162.

Fisher, Erik, und Arie Rip. 2013. Responsible innovation: Multi-level dynamics and soft intervention practices. In Responsible innovation. Managing the responsible emergence of science and innovation in society, Hrsg. Richard Owen, John Bessant und Maggy Heintz, 165-183. Chichester: Wiley.

Fisher, Erik, Roop L. Mahajan, und Carl Mitcham. 2006. Midstream modulation of technology: Governance from within. Bulletin of Science, Technology \& Society 26(6): 485-496.

Garud, Raghu, und David Ahlstrom. 1997. Technology assessment: A socio-cognitive perspective. Journal of Engineering and Technology Management 14(1): 25-48.

Geels, Frank W. 2011. The multi-level perspective on sustainability transitions: Responses to seven criticisms. Environmental Innovation and Societal Transitions 1(1): 24-40.

Geels, Frank W., und Johan Schot. 2007. Typology of sociotechnical transition pathways. Research Policy 36(3): 399-417.

Geels, Frank Walter. 2006. Co-evolutionary and multi-level dynamics in transitions: The transformation of aviation systems and the shift from propeller to turbojet (1930-1970). Technovation 26(9): 999-1016.

Gümüscü, Burcu. 2016. Lab-on-a-chip devices with patterned hydrogels. Engineered microarrays for biomolecule fractionation, organ-on-chip and desalination. Ph.D. thesis, University of Twente, Enschede. 
Guston, David H., und Daniel Sarewitz. 2002. Real-time technology assessment. Technology in Society 24(1/2): 93-109.

Hack, Lothar. 1995. TA als theoriegeleitete Interventionsstrategie. Der Ansatz des „Constructive technology assessment/CTA " in der sozialwissenschaftlichen Technikdebatte. Karlsruhe: Forschungszentrum Karlsruhe (Wissenschaftliche Berichte, FZKA 5641.

Hamaker-Taylor, Robin, Adriaan Perrels, Laura Canevari, Väinö Nurmi, Tuukka Rautio, Amanda Rycerz, und Francesca Larosa. 2018. Results of explorations of the CS market for the financial sector. EU-MACS deliverable 2.1. http://eu-macs.eu/wp-content/uploads/2019/02/EUMACS_ D21_FINAL.pdf. Zugegriffen am 02.12.2019.

Hennen, Leonhard, und Linda Nierling. 2014. Expanding the TA Landscape. Barriers and opportunities for establishing technology assessment in seven European countries. In Technology assessment and policy areas of great transitions proceedings from the PACITA 2013 conference in Prague, Hrsg. Tomáš Michalek, Leonhard Hennen, Constanze Scherz, Linda Nierling und Julia Hahn, 67-73. Prague: Technology Centre ASCR.

Héretier, Adrienne. 1993. Policy-Analyse. Elemente der Kritik und Perspektiven der Neuorientierung. Politische Vierteljahresschrift 24:9-38.

Hulshof, Frits. 2016. Topochip: Technology for instructing cell fate and morphology via designed surface topography. Ph.D. thesis, University of Twente: Enschede.

Jasanoff, Sheila. 2004. States of knowledge. The co-production of science and social order. London: Routledge.

Jasanoff, Sheila. 2005. Designs on nature. Science and democracy in europe and the United States. Princeton: Princeton University Press.

Kline, Stephen J., und Nathan Rosenberg. 1986. An overview of innovation. In The positive sum strategy: Harnessing technology for economic growth, Hrsg. Ralph Landau und Nathan Rosenberg, 275-305. Washington DC: National Academy Press.

Kuhlmann, Stefan, Patries Boekholt, Luke Georghiou, Ken Guy, Jean-Alain Héraud, Philippe Laredo, Tarmo Lemola, Denis Loveridge, Terttu Luukkonen, Wolfgang Polt, Arie Rip, Luis Sanz-Menendez, und Ruud E. Smits. 1999. Improving distributed intelligence in complex innovation systems. Final report of the advanced science \& technology policy planning network (ASTPP), a thematic network of the european targeted socio-economic research programme (TSER). Office for Official Publications of the European Communities. Karlsruhe: Fraunhofer ISI.

Kuhlmann, Stefan, Peter Stegmaier, und Kornelia Konrad. 2019. The tentative governance of emerging science and technology - A conceptual introduction. Research Policy (5): 1091-1097.

van Lente, Harro, und Jon van Til. 2007. A combined roadmapping-cluster approach for emerging technologies. International Journal of Foresight and Innovation Policy 3(2): 121-138.

Marris, Claire, Pierre-Benoît Joly, und Arie Rip. 2008. Interactive technology assessment in the real world. Dual dynamics in an iTA exercise on genetically modified vines. Science, Technology \& Human Values 33:77-100.

Parandian, Alireza. 2012. Constructive TA of newly emerging technologies. Stimulating learning by anticipation through bridging events. Ph.D. thesis, Technical University of Delft, Delft.

Parandian, Alireza, Arie Rip, und Haico Te Kulve. 2012. Dual dynamics of promises and waiting games around emerging nanotechnologies. Technology Analysis \& Strategic Management 24(6): 565-582.

Rip, Arie. 2011. Science institutions and grand challenges of society: A scenario. Asian Research Policy 2:1-9.

Rip, Arie. 2012. The context of innovation journeys. Creativity and Innovation Management 21(2): $158-170$.

Rip, Arie. 2018. Futures of science and technology in society. Wiesbaden: Springer VS.

Rip, Arie, und Haico Te Kulve. 2008. Constructive technology assessment and socio-technical scenarios. In Constructive technology assessment and socio-technical scenarios, Hrsg. Erik Fisher, Cynthia Selin und Jameson Wetmore, 49-70. Dordrecht: Springer. 
Rip, Arie, und Henk van den Belt. 1986. Constructive technology assessment: Influencing technological development? Journal für Entwicklungspolitik 3:24-40.

Rip, Arie, Thomas J. Misa, und Johan Schot, Hrsg. 1995. Managing technology in society. The approach of constructive technology assessment. London/New York: Pinter.

Rip, Arie. 2002. A co-evolutionary perspective on ELSI, CTA and other attempts at re-contextualisation of science and technology in society. Paper presented to the meeting of the European Association for the Study of Science and Technology, York, 31 July - 3 August 2002.

Robinson, Douglas K. R. 2010. Constructive technology assessment of emerging nanotechnologies. Experiments in interactions. Ph.D. thesis, University of Twente, Enschede.

Robinson, Douglas K. R., und Tilo Propp. 2008. Multi-path mapping for alignment strategies in emerging science and technologies. Technological Forecasting and Social Change 75(4): 517-538.

Schulze, Greiving, Verena, Douglas K. R. Robinson, und Séverine Le Gac. 2016. ,CTA-lite‘ for exploring possible innovation pathways of a nanomedicine-related platform - embedded responsible research and innovation in practice. In Responsibility and emerging technologies: Experiences, education and beyond, Hrsg. Diana M. Bowman, Anne Dijkstra, Camilo Fautz, Julia S. Guivant, Kornelia Konrad, Harro van Lente und Silvia Woll, 25-42. Berlin: AKA Press.

Smits, Ruud. 1992. Technikfolgen-Abschätzung in den Niederlanden mit besonderer Berücksichtigung der Niederländischen Organisation für Technikfolgen-Abschätzung (NOTA). In Technikfolgen-Abschätzung als Technikforschung und Politikberatung, Hrsg. Petermann, Bd. 1, 253-270. Karlsruhe: Veröffentlichungen der AFAS.

Stegmaier, Peter. 2009. The rock , $\mathrm{n}^{\text {' }}$ roll of knowledge co-production. EMBO Reports 10(2): 114-119.

Stegmaier, Peter, Stefan Kuhlmann, und Vincent R. Visser. 2014. The Discontinuation of sociotechnical systems as governance problem. In Governance of systems change, Hrsg. Susanna Borrás und Jakob Edler, 111-131. Cheltenham: Edward Elgar.

Te Kulve, Haico. 2011. Anticipatory interventions and the co-evolution of nanotechnology and society. Enschede: Ipskamp.

Te Kulve, Haico. 2014. Anticipating market introduction of nanotechnology-enabled drug delivery systems. In Application of nanotechnology in drug delivery, Hrsg. A. Demir Sezer, 501-524. London: IntechOpen.

Te Kulve, Haico, und Kornelia Konrad. 2017. Sectoral demand articulation: The case of emerging sensor technologies in the drinking water sector. Technological Forecasting and Social Change 119:154-169.

Turnheim, Bruno, und Frank W. Geels. 2012. Regime destabilisation as the flipside of energy transitions: Lessons from the history of the British coal industry (1913-1997). Energy Policy 50:35-49.

Van de Poel, Ibo. 2000. On the role of outsiders in technical development. Technology Analysis \& Strategic Management 12(3): 383-397.

van Merkerk, Rutger O. und Douglas K. R. Robinson. 2006. Characterizing the emergence of a technological field: Expectations, agendas and networks in Lab-on-a-chip technologies. Technology Analysis \& Strategic Management 18(3-4): 411-428.

Vergragt, Philip, und Peter Groenewegen. 1989. New technological development and technology assessment: A plea for an integrated research. PROJECT Appraisal 4(1): 29-35.

Visscher, Klaasjan. 2020. Theatrical technology assessment. A role-play simulation for bridging the gap between technology and society in interdisciplinary engineeing education. Science, Technology \& Policy Studies - STePS Working Paper Series 01/20.

Visscher, Klaasjan, Peter Stegmaier, Andrea Damm, Robin Hamaker-Taylor, Atte Harjanne, und Raffaele Giordanao. 2020. Climate services. Matching supply and demand: A typology of climate services. Climate Services 17:100136.

Wynne, Brian. 1975. The rhetoric of consensus politics: A critical review of technology assessment. Research Policy 4:108-158. 